\title{
The Utilization of Signal Analysis by Using Short Time Fourier Transform
}

\author{
Indriati Retno Palupi ${ }^{1}$, Wiji Raharjo ${ }^{2}$ \\ 1,2 Geophysical Engineering, UPN “Veteran” Yogyakarta
}

\begin{abstract}
Signal Analysis is a part of geophysics work. It is important in analyze the character of signal or waveform in geophysics. In this paper the earthquake waveform is used as the example. One method to do this is used Short Time Fourier Transform. It adopts the basic concept of Fast Fourier Transform in the short period of time in waveform and at the same moment there is a convolutional process between the waveform and the mother wavelet and then resulting the spectrogram. Finally, the spectrogram will show the power spectrum or the magnitude of the amplitude in each time in the waveform. It relates with the energy of the earthquake. The result including three parameters, they are time, frequency and the spectrogram. It makes easier for the geophysicist to analyze the frequency changing in each time based on the spectrogram colour. Besides that, it can be used to identify the arrival time of $\mathrm{P}$ and $\mathrm{S}$ wave as the important information in calculate the hypocentre location of the earthquake.
\end{abstract}

Keywords: Waveform, earthquake, Signal Analysis and Short Time Fourier Transform

This is an open access article under the CC-BY-NC license

\section{INTRODUCTION}

There are many physics natural phenomena recorded and visualized as the signal or waveform. For example, the earthquake, it is a transfer energy caused by tectonic plate's movement to the whole area. The vibration coming from the earthquake, recording by the seismometer and then visualized like the aperiodic signal or waveform. Actually, the signal brought some information like the amplitude or the energy of the wave, period and also the time. Signal analysis in geophysics has an important rule to describe the waveform phase characteristic. Phase in the waveform can give the description about hypocentre location. It is very important in disaster mitigation.

Generally, there are two phase that should be known of the earthquake waveform. They are $\mathrm{P}$ and $\mathrm{S}$ phases. Both of them include to the body wave. They have certain frequency, and knowing the arrival time for $\mathrm{P}$ and $\mathrm{S}$ is an initial input to calculate the hypocentre location. As we know that the waveform is in the time domain, so the first thing to do, the waveform should be converted to the frequency domain by the Fast Fourier Transform (FFT) method. If the frequency domain include to the P and S category, then we can pick their arrival time. However, pick the $S$ phase is more difficult than the $P$ phase because it is usually contaminated by the noise or the P phase coda. To solve the problem applied some filters is important to neglect the noise before picking process (Garcia et al., 2016).

After pick the $\mathrm{P}$ and $\mathrm{S}$ phases of the waveform from minimal three stations, then the origin time is calculated by using Wadati Method. Then, the epicentre location is calculated by three circle method where we should calculate the radius of each station to get the intersection of them. And finally, the hypocentre is calculated by Pythagoras Method based on one stations data. Anyway, for the epicentre, it can be calculated by single station data, but the result is worse than 3 stations.

However, FFT just gives information about the frequency of the waveform. It does not give the information about the frequency chancing to the time (Lopez, 2000). It works well for knowing the P phase arrival time, but sometimes not good in the $\mathrm{S}$ phase. It can be caused from the noise factor that usually include in the waveform. The other method is Short Time Fourier Transform (STFT). It is similar with the FFT method. It works in the shorter "window" than the FFT, and at the same time the waveform 
is convolving with the mother wavelet so we can see the changing of the frequency to the time in the spectrogram.

\section{RESEARCH METHOD}

Fast Fourier Transform (FFT) is the general method in analyse the signal or waveform. When FFT applied to the waveform, the time domain is lost and converting to the frequency domain. Then the information of the wavefom is just only the frequency. To pick the P and S phases we should go back to the original waveform and it is not an effective works (Colak et al., 2016). In the FFT methode, the waveform is assumed as the sinusiodal waveform. The first way, the waveform is devided into sinus and cosinus wave. The second way, FFT applied the euler equation of trigonometry to the waveform. As we know that the euler is the summation of the sinus and cosinus wave (Heckbert, 1998). Generally, STFT adopts the FFT method in the smaller window size to get the $P$ and $S$ wave arrivaltimes.

$P$ phase is always recorded first. It has bigger velocity value and shorter amplitude value than the $\mathrm{S}$ phase. Amplitude in the waveform representing how much the energy is absorbed by the medium. Indirectly, it can be a sign the medium condition. The higher of amplitude value in the waveform is interpreted that the medium (weak) absorbing the energy more than the shorter amplitude (rigid) (Bormann, 2014). The example of the waveform and the FFT can be seen in Figure 1, while the example of the STFT can be seen in Figure 2

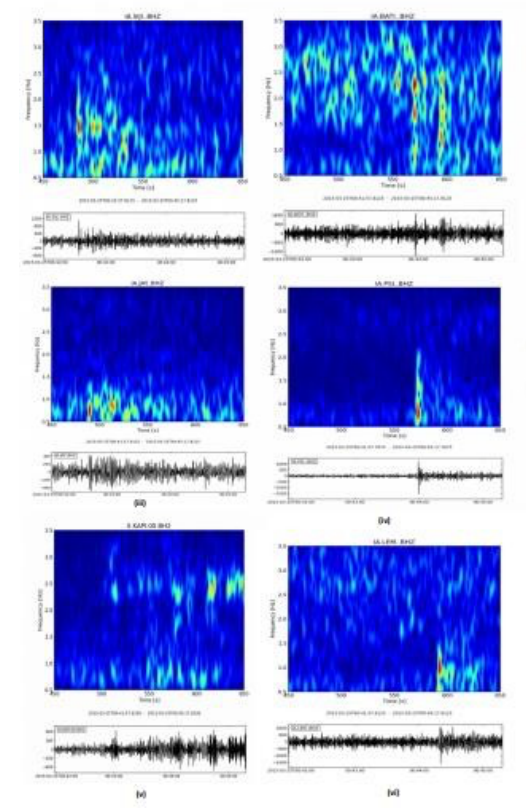

Figure 1. The waveform (upper) and its FFT result (lower) [2]
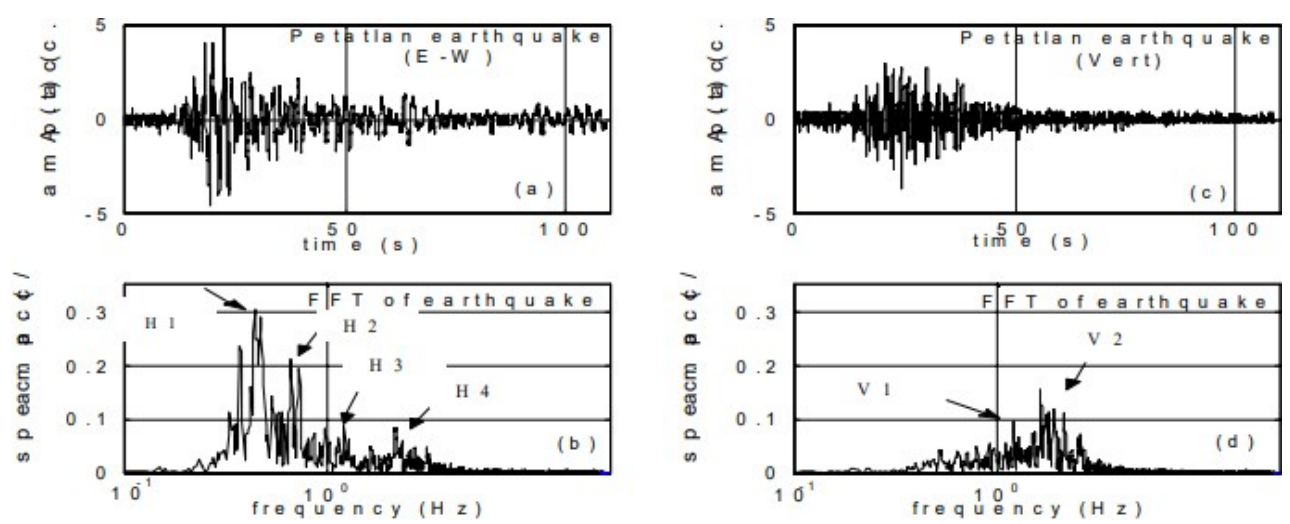

Figure 2. The STFT of Hokaido earthquake [6] 
STFT is defined by the equation

$$
x_{i}[m]=x[m+l H] w[m]
$$

Where $\mathrm{x}$ is the waveform, $\mathrm{m}$ is the time function, $\mathrm{H}$ is the total sampling of the length window (l) and $w$ is the window function. To simplify the calculation, discretization of equation (1) is done like in the equation (2)

$$
X[k, l]={ }_{M} \bar{\sum}_{m=1} x_{i}[m] e^{-j 2 \pi^{m k}}
$$

Finally, the spectrogram can be calculated by the equation

$$
\operatorname{spec}=\frac{2}{\sum_{\substack{M \\ m=1} \frac{w[m]}{N}}^{M}}|\dot{X}[k,]|
$$

The Spectrogram give more details in frequency and time of the waveform. Like in Figure 2, there are colour represent the changing of the frequency to the time. The $\mathrm{x}$ and $\mathrm{y}$ axis are the time and the frequency. $\mathrm{P}$ and $\mathrm{S}$ phasesof the waveform can be identified well from the two parameters (frequency and the time) at the same time (Zhimorimov, 2019). Furthermore, the $\mathrm{P}$ and S phases analysis can be used to litosphere interpretation. Their amplitude is describing the rigidness of the medium. Besides that, the time span between their arrival time is also describe the type of the earthquake (Sato et al., 1997). STFT is also be a basic method to the wavelet transform analysis that visualizing the time and the frequency in the same moment by the convolutional process. If a mother wavelet with certain scale is applied to the waveform, it will result a certain pattern of the scalogram. The scale acts like the window in FFT or STFT (Sinha it al., 2015)

\section{RESULT AND DISCUSSION}

The data (earthquake waveform) is downloaded from the IRIS website (Iris, 2021). IRIS provide the catalogue and also the waveform data of the earthquake in the world. We choose earthquake in Aceh in 2004 and Lombok in 2018 as the input data. The earthquakes are include to the biggest disaster phenomena in the world. Their magnitude are more than 9 SR and 6 SR and causing many fatalities. The waveform of earthquake in Aceh can be seen in Figure 3.

Figure 3 below shows the waveform of earthquake in Aceh in 2004 and its FFT result. We can see that the frequency of the earthquake is reach to $11 \mathrm{~Hz}$ (the value in the left top of the Figure 3). It is included to the tectonic earthquake phenomena because it is happened from the movement of Australian plate under the Eurasian plate in subduction zone in the west of Aceh.

We use the FFT to select the waveform based on its frequency. It is good to get the information about the tectonic earthquake. Then the waveform is cutted before it goes to the STFT process. In STFT there is a convolutional process between the waveform and the mother wavelet. 


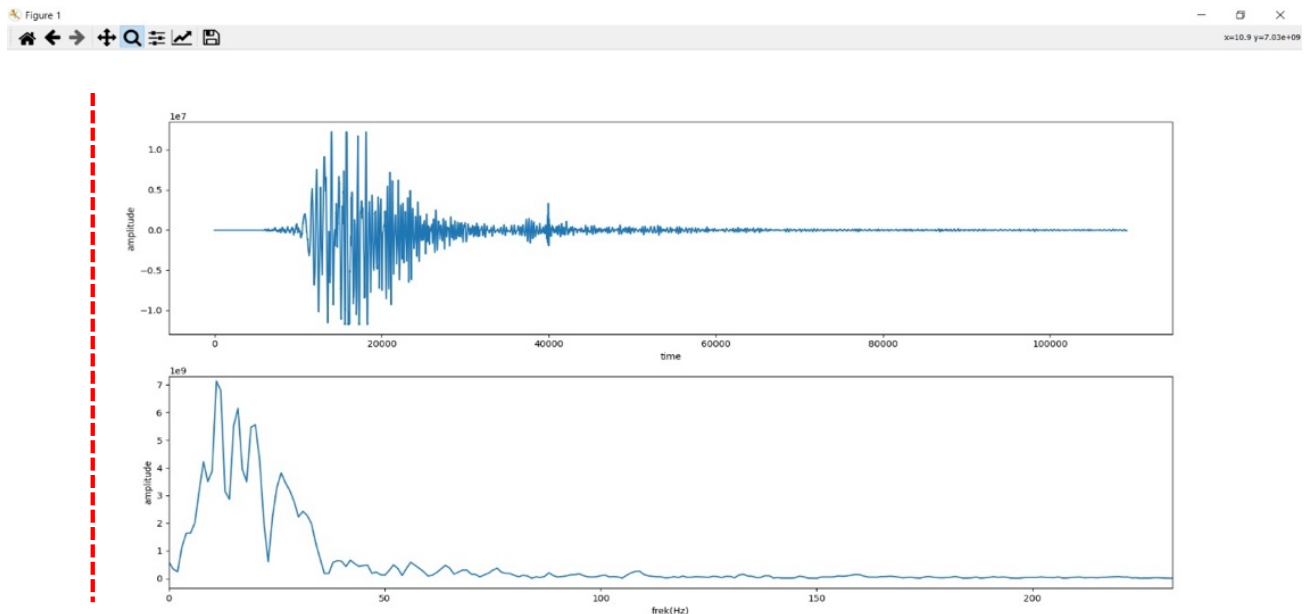

Figure 3. Aceh earthquake waveform (upper); the FFT result (lower)

The convolutional process determines the size of the spectrogram visualization. Every "point" in the waveform is multiplied with each "point" in the motherwavelet. Finally, the spectrogram will have N x M size of matrix, where $\mathrm{N}$ and $\mathrm{M}$ are coming from the number of waveform and the motherwavelet data (Saachi, 2002).

P and S phases are determined by STFT method. The description of STFT result is in Figure 4.

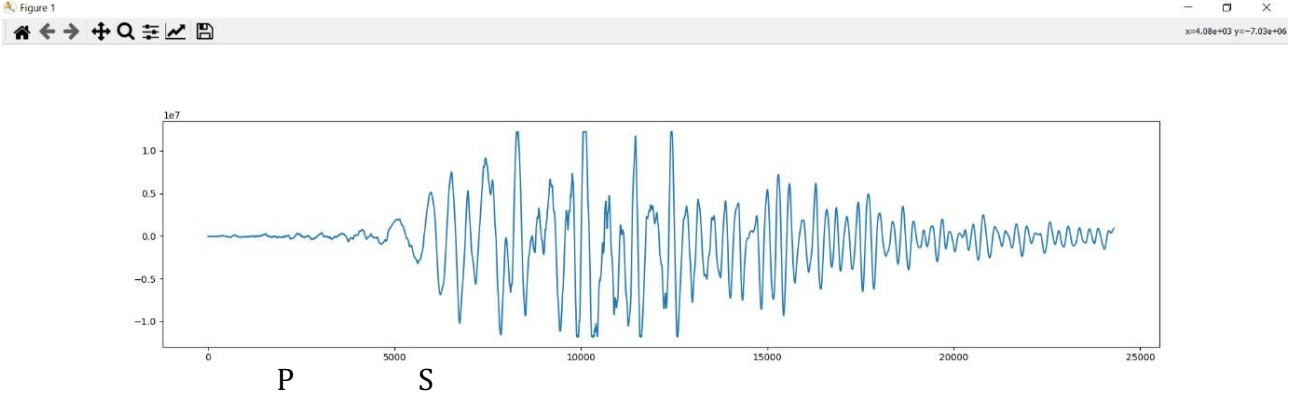

Figure 4. The waveform of earthquake in Aceh 2004 (upper); the STFT result (lower)

The STFT shows the changing of the frequency to the time based on the spectrum. The colour representing the magnitude of the waveform. As we can see in the scale bar, the high spectrum value is represented by red colour and the low value with the blue one. The spectrum has good correlation with the amplitude. In Figure 4, before P phase, the amplitude almost reach to zero (blue colour). While in the $P$ phase, the spectrum begin shows its magnitude and the $P$ phase arrival time is identified. The same rule in identifying the $\mathrm{S}$ phase, where it has higher amplitude than the $\mathrm{P}$ phase, the magnitude spectrum will show the higher value/colour.

While, Lombok earthquake waveform has different characteristic to the Aceh earthquake waveform. As the natural phenomena, the waveform character will be different between one area and the others. Aceh located in Sumatera, one of the big island in Indonesia. While, Lombok is a little one that located next to Bali. Both of them are face to the subduction zones. The subduction zone faces to Lombok is similar with Java Island, perpendicular direction relatively. Meanwhile, subduction zone faces to Aceh has a certain angel. The effect of the faces of the subduction is the shaking level of the magnitude value. Java and Lombok citizens feel the bigger shock for the 5 magnitude of earthquake than Sumatera citizens relatively. The perpendicular subduction face of Java and Lombok cause the maximal forcetransfer.

Lombok earthquake in 2018 was started from the foreshock that was rarely happened in Indonesia. There were some noises in Lombok earthquake. Different with the Aceh earthquake, the 
waveform is good well, almost clean from the noise. While, the Lombok earthquake has noises, foreshcok, mainshock and aftershock waveform. The Lombok earthquake waveform (mainshock) can be seen in Figure 5

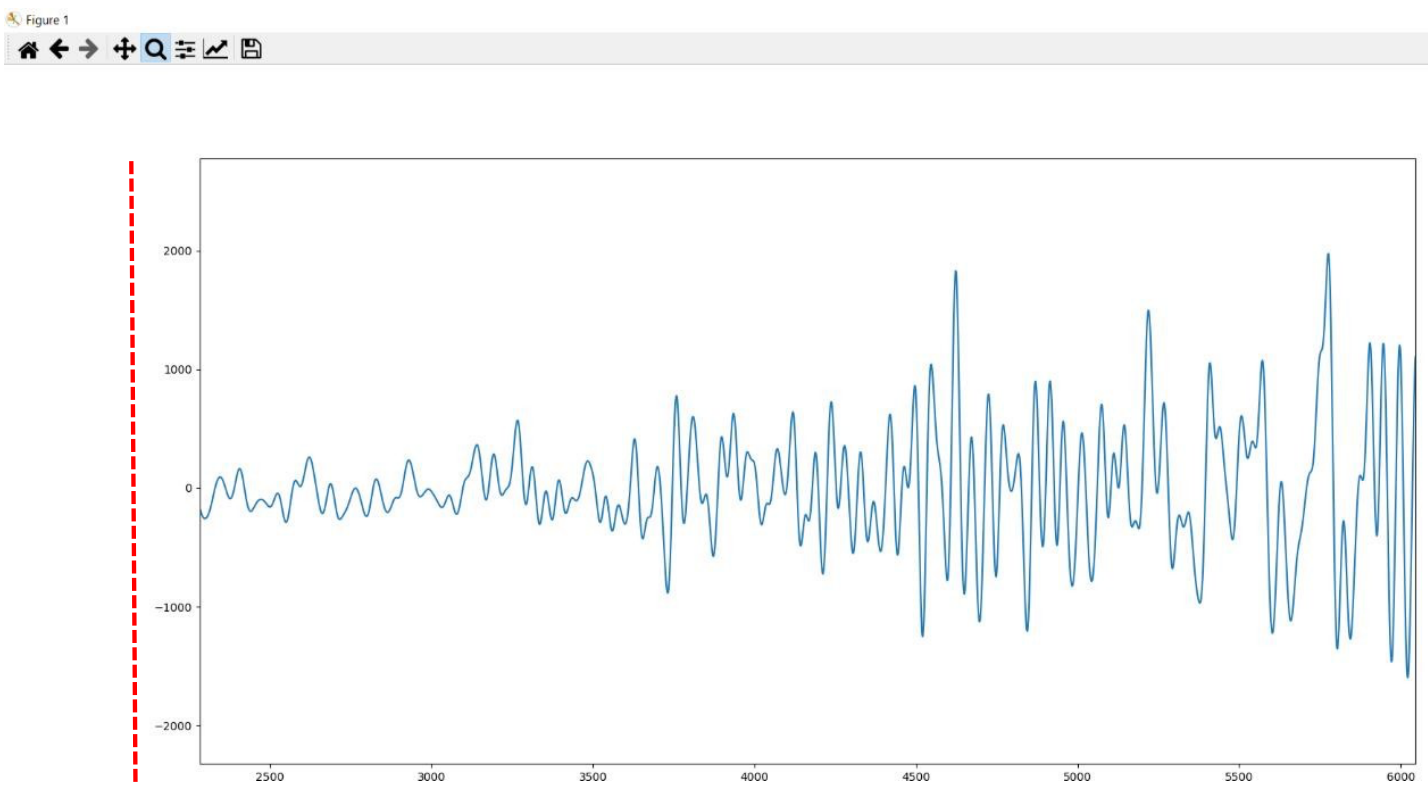

Figure 5. Lombok earthquake waveform

Based on FFT calculation, the Lombok earthquake waveform frequency is $29 \mathrm{~Hz}$, like in Figure 6.

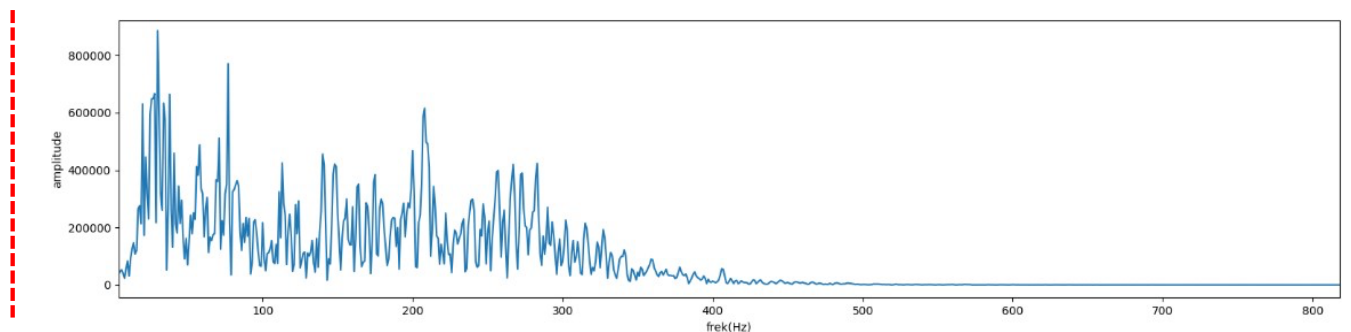

Figure 6. FFT result of Lombok earthquake

From the Figure 5, we can see that the noise is recorded in the Lombok earthquake waveform (circle blue). It will affected to the FFT and STFT calculation. Removing the noise can be done by the filter method. Generally, there are three filters, they are low pass, band pass and high pass filter. Low pass filter enhances the low frequency and neglected the high frequency. Band pass filter enhances certain frequency band where the value is determined by the user. And the high pass filter enhances high frequency and neglected the low frequency of the waveform. To make the decision which one the filter should be used, trial and error process is needed.

STFT method is applied to the Lombok earthquake waveform after the FFT calculation. With the same way like in Aceh earthquake waveform, the STFT of Lombok earthquake more varies than Aceh earthquake. It can be seen in Figure 7. 


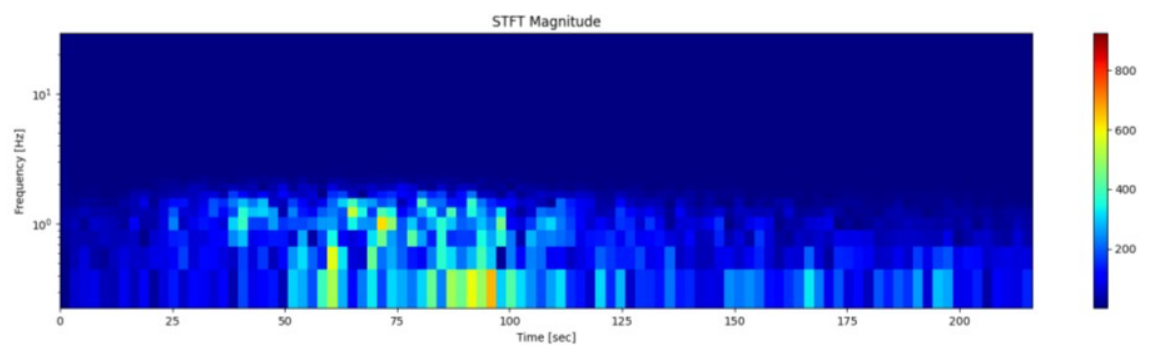

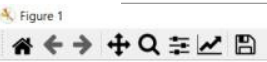

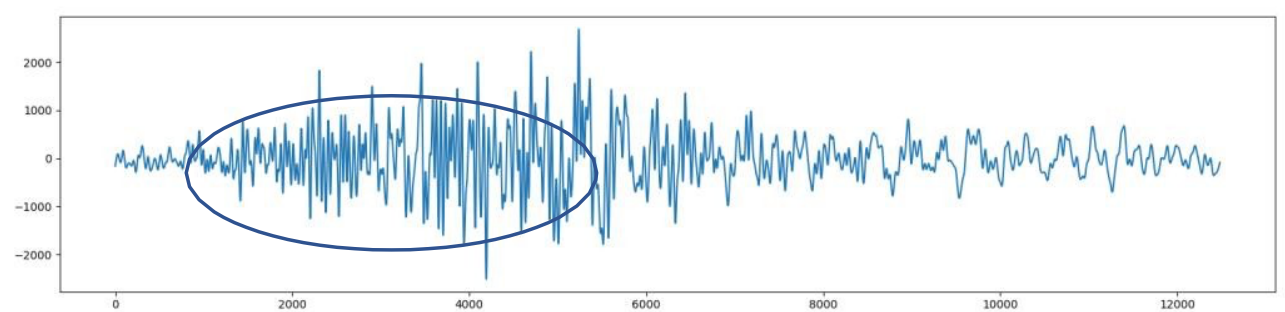

Figure 7. STFT result of Lombok earthquake waveform

Based on Figure 7, it is more difficult to make the decision of $\mathrm{P}$ and $\mathrm{S}$ phases' arrival time. The STFT shows that the pattern of the spectrogram is more varies than Aceh earthquake waveform although the magnitude of Lombok earthquake is smaller. Similar with STFT result of Aceh earthquake waveform, the blue and red colours are identic with the low and high magnitude of the power spectrum. Again, the spectrogram make the P and S phase's arrival time becoming easier. Although the P phase colour in power spectrum reach to the low magnitude, but as we can see in the y axis, it is still has big value. The red dashed lines in figure 7 show the $P$ and $S$ phases' arrival time. The $S$ phase is showed by the brighter colour in the spectrogram. Due to the power spectrum scale bar, it is included to the higher value of the amplitude of the waveform.

If we make a comparison of STFT result between Aceh and Lombok earthquake waveform, the Aceh earthquake waveform is more dens than the Lombok earthquake waveform. It can be caused by two factors for general. First, the magnitude of the earthquake, Aceh has the bigger one (indeed it is still the biggest one magnitude in Indonesia). The other factor is the period of the waveform. Aceh is included to the long period. Long period waveform usually has more dens pattern of power spectrum. However, the depth of the earthquake should be includeed to the parameter. The earthquake with the big magnitude but its location is very deep sometimes resulting the low magnitude in the waveform. It is caused by the absorbing the energy of the earthquake along the medium. Otherwise, the medium magnitude but it is included to the shallow earthquake can be a big disaster like in Lombok and it will resulting high amplitude (high power spectrum also).

The spectrogram value depends on the amplitude, it means that the spectrogram is a vizualization of the energy of earthquake (Johnson, 1989). The spectrogram is also called the power spectrum density because it visualizes the dense of the waveform based on the waveform characteristic (Darnilla, 2018). The goals of the waveform charasteristic analysis is the hypocentre location. Knowing it is similar with knowing the source of the vibration of earthquake (Reynolds, 2011) and the elasticity of the medium (Lowrie,2007).

\section{CONCLUSION}

STFT is one method to identify P and S phase of the earthquake waveform. It is important to do because they are an input data in calculating the hypocentre location. STFT shows the P and S wave based 
on its spectrum that coming from its calculation. It calculates the frequency like in FFT method, while at the same time, it convolves the waveform with the mother wavelet resulting the spectrogram. The spectrum then shows the frequency changing to the time. Finally, by STFT method, the characteristic of the $\mathrm{P}$ and $\mathrm{S}$ phase can be known together with their arrival time.

\section{REFERRENCE}

Bormann, Peter., Klaus Klinge dan Siegfried Wendt. 2014. Data Analysis and Seismogram Interpretation. DOI : $10.2312 /$ GFZ.NMSOP-2_ch11 2014

Colak, Omer H., Tahir C Destiel, Sukru Ozen, Hasan Arman, Osman Cerezel. Detection of P and S Wave Arrival Times Using The Discrete Wavelet Transform in Real Seismograms. The Arabian Journal for Science and Engineering Volume 34 Number $1 A 2016$

Darnilla Eva., Mutammimul Ula, Kerista Tarigan, Tonni Limbong, Marzuki Sinambela. Waveform Analysis of Broadband Seismic Station Using Machine Learning Python Based on Morlet Wavelet. IOP Conference Series : Material Science and Engineering 2018

Garcia, Luz., Isaac Alvarez, Carmen Benitez, Manuel Titos, Angel Bueno, Sonia Mota, Angel de la Torre, Jose C Segura, Gerardo Alguacil, Alejandro Diaz-Moreno, Janire Prudencio, Araceli Garcia-Yeguas, Jesus M Ibanez, Luciano Zucarello, Ornella Cocina Domenico Patane. Advances On the Automatic Estimation of the P-wave Onset Time. Annals of Geophysics 59, 4, 2016

Heckbert, Paul. Fourier Transform and The Fast Fourier Transform (FFT) Algorithm. Notes, Computer Graphics 2, 15-463 1998

Johnson, Lane R and T V McEvily. Near-Source of Quarry Explossions. Proceeding of The 11th Annual DARPA/AFCL Seismic Research Symposium 1989

Lopez, Carlos I Hueta., Yong June Shin., Edward J Powers and Jose M Rosset. Time-Frequency Analysis Of Earthquake Records. USA : 12WCEE 2000

Lowrie, William. Fundamentals of Geophysics. Cambridge University Press 2007

M D Saachi. Statistical Transform Methods in Geophysical Signal Processing. Department of Physics University of Alberta 2002.

Reynolds, John M. An Introduction to Applied and Environmental Geophysics. Willey Blackwell 2011

Sato, Haruo and Michael C Fehler. Seismic Wave Propagation and Scattering in the Heterogenous Earth. Springer-Verlag Berlin Heidelberg, 1997

Sinha, Satish., Partha Routh, Phil Anno John Castagna. Spectral Decomposition of Seismic Data with Continous Wavelet Transform. https://www.researchgate.net/publication/268206177 2015

Wibowo B., N Hendaryoko and S Rohadi. Preliminary Study of the CTBTO's Seismic Station Characteristic Using Spectral Analysis Method in Indonesia. BMKG

Zhimorimov, Hristo. 2019. On the Development of STFT-analysis and ISTFT-synthesis 